\title{
Machine learning under the spotlight
}

Zibar, Darko; Wymeersch, Henk; Lyubomirsky, Ilya

Published in:

Nature Photonics

Link to article, DOI:

10.1038/s41566-017-0058-3

Publication date:

2017

Document Version

Peer reviewed version

Link back to DTU Orbit

Citation (APA):

Zibar, D., Wymeersch, H., \& Lyubomirsky, I. (2017). Machine learning under the spotlight. Nature Photonics, 11(12), 749-751. https://doi.org/10.1038/s41566-017-0058-3

\section{General rights}

Copyright and moral rights for the publications made accessible in the public portal are retained by the authors and/or other copyright owners and it is a condition of accessing publications that users recognise and abide by the legal requirements associated with these rights.

- Users may download and print one copy of any publication from the public portal for the purpose of private study or research.

- You may not further distribute the material or use it for any profit-making activity or commercial gain

- You may freely distribute the URL identifying the publication in the public portal

If you believe that this document breaches copyright please contact us providing details, and we will remove access to the work immediately and investigate your claim. 
Machine learning under the spotlight

Darko Zibar, Henk Wymeersch and Ilya Lyubomirsky

\section{The field of machine learning potentially brings a new set of powerful tools to optical communications and photonics. However, it is vital that such tools are evaluated properly and used judiciously.}

Recently, there has been an increasing amount of research focused on the application of machine learning techniques to optical communication. These applications have varied from component characterization, performance prediction and system optimization, and more recently, within the field of quantum communication and optical fibre sensing. The question that remains to be answered is whether the application of such schemes is one of hype with limited real impact, or whether it can truly bring significant advantages with orders of magnitude improvement compared to the conventional methods and the benefit of reduced human involvement.

To answer those questions, we organized a workshop at the 2017 European Conference on Optical Communication (ECOC) in Gothenburg, Sweden, with the title: Opportunities for machine learning in optical communication: from components characterization, systems design and network optimization. In addition to the annual Optical Fibre Communication Conference (OFC) in the US, ECOC is arguably most important and influential conference in the field. Typically, the topics of the workshops are carefully selected to reflect the latest trends and possible future paths of the field.

This workshop was divided in three parts; the first part was focused on the application of machine learning techniques to physical layer, the second part was oriented towards the application of machine learning techniques to networking layers. Finally, the third part was focused on the non-telecom applications, but still related to photonic technologies.

Before describing the contents of the workshop in more detail, we would like to review why machine learning is potentially useful and beneficial to the field of optical communication, and why it is attracting such strong attention from the community. As described in the Workshop's keynote talk by Thomas Schön from Uppsala University, one example when machine learning can be very useful is when the relationship between the input and the output of the system is complex and intractable, or to express it differently when there is a lack of a model describing the relation between the input and the output of the system. Techniques from machine learning can then be used to learn an effective model from the acquired data, and then use the inferred model for optimum signal detection and performance prediction. Advances in deep learning, in combination with high-performance computing and easy-to-use software interfaces, have made such approaches accessible to 
anyone. Moreover, machine learning techniques are also attractive to use when it is desirable to learn the nonobservable time-varying dynamics from the detected data such as: the evolution of amplitude and phase noise, polarization mode dispersion, nonlinear interference and photon density.

It should also be emphasized that in some cases even though the system is difficult to describe using conventional approaches, approximate analytical models can be derived. The derived model can indeed be very useful to provide prior knowledge of the system and accelerate learning (less data is needed to learn the model). These principles can be applied at different layers, ranging from the device-level to large-scale networks, whenever the system exhibits complex behavior that is hard to model and optimize. For that reason, machine learning can serve as a useful tool, complementing and possibly replacing current methodologies.

Some specific examples in optical communication that have already benefited from machine learning techniques are: optimum signal detection of data transmitted over a nonlinear fibre optic channel; and performance prediction (in terms of error rate and reach) of wavelength division multiplexed optical communication systems. Other successful tasks include prediction of chromatic dispersion, polarization mode dispersion, polarization dependent loss, optical signal to noise ratio, modulation format and baud rate from the detected signal power levels without looking into transmitted data sequence. In addition, other achievements are the prediction of outage in optical networks and finally extraction of intensity and frequency noise of nanoscale components such as nano-lasers.

During the first session of the workshop Alan P. T. Lau from the Hong Kong Polytechnic University gave a detailed overview of some of the above-mentioned topics and also presented his group's latest work in this area. Hansjoerg Haisch from Keysight Technologies also gave his vision as to how machine learning and data analytics may impact future business models for the test and measurement industry. This was followed by talks from Takahito Tanimura from Fujitsu Labs and Seb Savory from Cambridge University describing the application of deep and shallow neural networks for optical performance monitoring. Tanimura discussed deep neural networks and reported accurate optical-signal-to-noise ratio estimation, irrespective of the baud rate and modulation format, from the samples right after digital coherent detection (prior to signal demodulation). Seb Savory described how neural networks make it possible to distinguish and estimate noise associated with amplified spontaneous emission and nonlinearities (nonlinear noise) simultaneously.

However, neural networks only provide a point estimate (mean value of the quantity of interest, i.e. signal-tonoise ratio, error rate, optical fibre channel parameters and nonlinear interference) and for optical network design and optimization having knowledge of the uncertainty around the mean value (confidence interval) is very useful for determining the margins of network operation. Generally speaking, margins can be used to reduce network over-dimensioning. Jakob Thrane from the Technical University of Denmark described how a machine learning technique called Gaussian Process Regression (GPR) can be used to provide mean value and the uncertainty measure $(95 \%$ confidence interval) for optical performance monitoring of WDM communication systems. GPR was used first to learn the complex relationship between the system design parameters (transmission power, number of spans, channel spacing and symbol rate) and the error rate, and then later predict the error rate for a new set of design parameters. Finally, a talk by Sebastian Kleis from 
Helmut Schmidt University in Hamburg, demonstrated how particle filtering, which is a Bayesian filtering technique, can be used to enable phase noise compensation for a continuous-variable quantum key distribution link.

The second part of the workshop focused on the application of machine learning at the networking layer, mainly in the form of data analytics, which describe, explain, predict, and prescribe network operations. Danish Rafique from ADVA Optical Networking highlighted that, with increasing costs of operating network and diminishing revenue, data analytics can reduce operational expenses, improve user experience, and predict weaknesses and faults. This is because, through machine learning, fully automatic network design and maintenance becomes possible. Two academic talks focused on specific ways in which machine learning can improve the networking layer. Cristina Rottondi from the Dale Mole Institute of Artificial Intelligence advocated the use of machine learning to dynamically determine preferential routing and light-path configurations. If faults or degradations do occur in an optical network, Luis Velasco from Polytechnic University of Catalonia suggested the use of a machine learning architecture to monitor networks, detect and localize faults, and finally adapt the network, all without human intervention. Finally, Naga Vishnu Kanth Irukulapati from Ericsson Research presented a vision for applying machine learning to future communication infrastructure. With the advent of 5G, there will be the production of massive amounts of data, which can be leveraged in self-learning systems. In the final part of the workshop, Peter Bienstman of Ghent University presented his research on an integrated photonics implementation of reservoir computing neural networks. Finally, Satyajeet Sing Ahula of Facebook gave a fascinating view of machine learning applications in Facebook's datacenters.

The workshop demonstrated that field of machine learning brings a new set of powerful tools to the optical communication and photonics communities. However, it is clear that great care must be taken when applying these tools. For instance, in the case of signal transmission in the linear regime, optimum detection techniques already exist and it is hard to justify why machine learning tools should be used. Hence, machine learning should not be applied simply to problems where it is easy to do so, but rather where it brings significant quantitative benefits, either in performance, reduction of human involvement during design or deployment, automation, or adaptation abilities. In turn, this implies that any application of machine learning should be measured in those metrics and benchmarked against the state of the art.

Looking to the future, we believe that there are more situations in photonics in general that may benefit from machine learning techniques. Progress in optical communications is very hardware driven. As new technologies appear, the first approach will likely still be to use conventional modeling and when models become too cumbersome, machine learning can replace them. Moreover, for future next-generation optical communication systems employing multi-core, multi-mode fibres and wavelength division multiplexing deriving suitable models is very challenging due to a large number of parameters and complex interactions. As a result, machine learning techniques may prove useful to learn those models. For instance, deep neural networks may be an interesting area to explore for accurate modeling and compensation of nonlinear effects in this type of optical transmission system. 
Ultimately, a hierarchy of machine learning approaches may co-exist, optimizing networks from the device to network level. At the same time, the introduction of machine learning brings the risk that less effort gets spent on understanding fundamental research and that may become a crutch that will do the work for us, providing little or no insight.

To mention some more specific future applications of machine learning, it can be used to capture laser dynamics and parameters which are difficult to model by standard approaches. A significant advantage compared to the traditional methods is that a single measurement is needed to fully characterize the laser and additionally, the uncertainty (confidence interval) of the relative intensity and frequency noise measurement, is directly obtainable. Another advantage is that such techniques work well at low signal-to-noise ratio which is the case for quantum communication links and nano-lasers. Finally, an unexplored application of machine learning techniques is the field of optical fibre sensing. Typically, the state-of-the-art optical fibre sensors require a certain amount of pre-processing to compute the desired parameters such as strain and temperature. It would be beneficial to be able to learn directly the relationship between the raw data and the measurement parameters, predict evolution of temperature and strain, and finally get an estimate of the uncertainty.

In conclusion, it is too soon to say whether machine learning will solve the most important and pressing problems in the field of optical communications. Nevertheless, the workshop indicates that there are certain promising applications, where machine learning can solve specific problems that cannot be solved by conventional approaches. Currently, many machine learning techniques are not well known in the photonics community. However, we believe that with time they will slowly become common knowledge and widely applied in the field. We hope that in the near future, courses on machine learning will be an integral part of the electrical and photonics engineering curricula and that applying machine learning to a given problem will become as common as applying numerical simulations.

Darko Zibar, DTU Fotonik, Technical University of Denmark, dazi@fotonik.dtu.dk Henk Wymeersch, Chalmers University of Technology, Sweden, henkw@chalmers.se Ilya Lyubomirsky, Inphi, USA, ilyubomirsky@inphi.com 


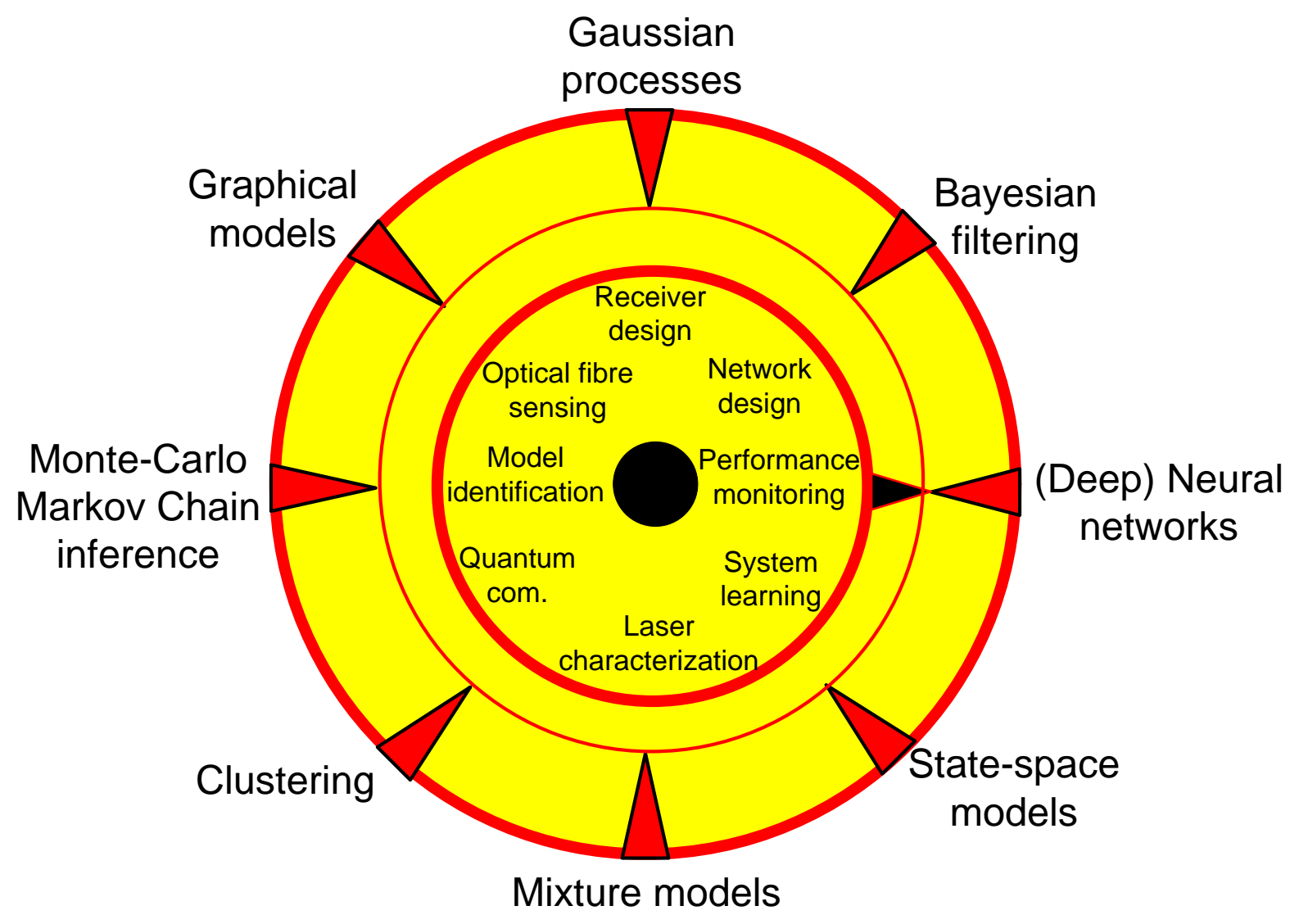

Figure 1: Illustration of different machine learning techniques and applications where they may have impact. Credit: Darko Zibar. 